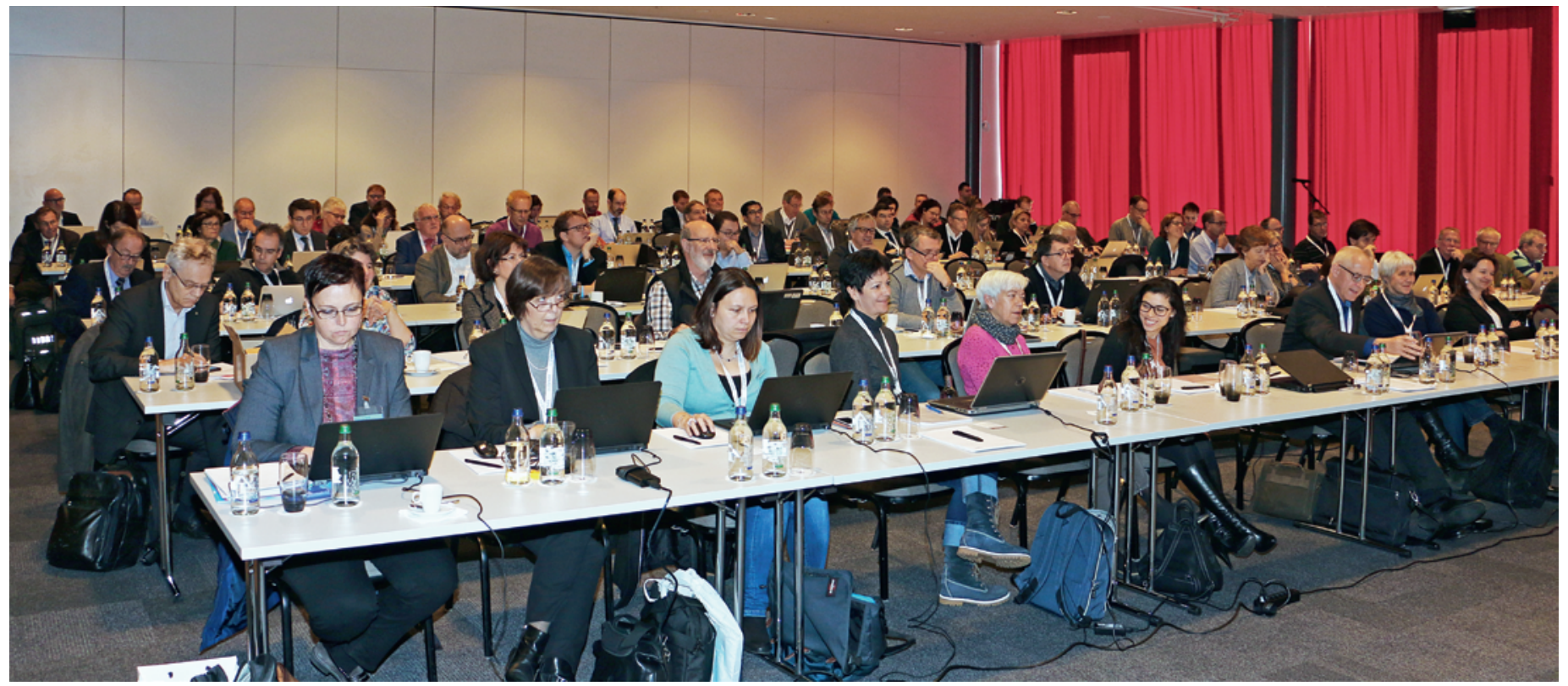

Hôtes et délégués ont été largement informés en plénum des champs d'action de I'ISFM.

\title{
Des enjeux et défis très divers
}

\section{Bruno Kesseli}

Dr méd. et lic. phil., rédacteur en chef

Le domaine de la formation postgraduée et continue est en pleine évolution, c'est du moins la conclusion que l'on peut tirer de l'assemblée plénière annuelle de l'ISFM dont plusieurs points à l'ordre du jour ont donné lieu à des discussions animées.

Après ses mots de bienvenue adressés aux délégués et aux hôtes, Werner Bauer a présenté une vue d'ensemble des tâches permanentes de l'ISFM et un flash d'information soulignant les nombreuses sollicitations auxquelles son Institut doit actuellement faire face. Le sens de l'efficacité et de la diplomatie dont font preuve le président de l'ISFM et ses collaborateurs et collaboratrices pour y répondre n'a cessé d'être mis en avant tout au long de la journée.

\section{L'infoflash souligne la diversité}

L'ISFM est chargé de la formation postgraduée de plus de 10000 médecins-assistants dans près de 1500 établissements de formation et de 1000 cabinets formateurs. Chaque année, il accomplit plus de 100 visites d'établissements et traite des milliers de demandes de titre ou de plans de formation. Il édicte les réglementations de la formation postgraduée et de la formation continue (RFP, RFC), élabore, actualise et veille à la mise en application de pas moins de 117 programmes de formation, pour ne citer que quelques-unes de ses activités récurrentes.

\footnotetext{
Assemblée plénière de I'ISFM

Organe autonome de la FMH, I'Institut suisse pour la formation médicale postgraduée et continue (ISFM) réunit tous les acteurs et organismes principaux du domaine de la formation médicale postgraduée et continue, et garantit aux médecins une formation de qualité élevée dans plus de 120 domaines spécialisés.

L'assemblée plénière annuelle de I'ISFM remplit différentes fonctions. D'une part, les membres du plénum forment un Comité doté d'un pouvoir de décision et d'élection, qui traite les affaires relevant de son domaine de compétences à l'instar de la Chambre médicale de la FMH. De l'autre, I'assemblée, à laquelle sont également conviés des invités de provenances diverses, constitue une plate-forme chargée de transmettre des informations sur des thèmes spécifiques, de garantir l'échange d'expérience entre les participants et de débattre de toutes sortes de sujets.
} 


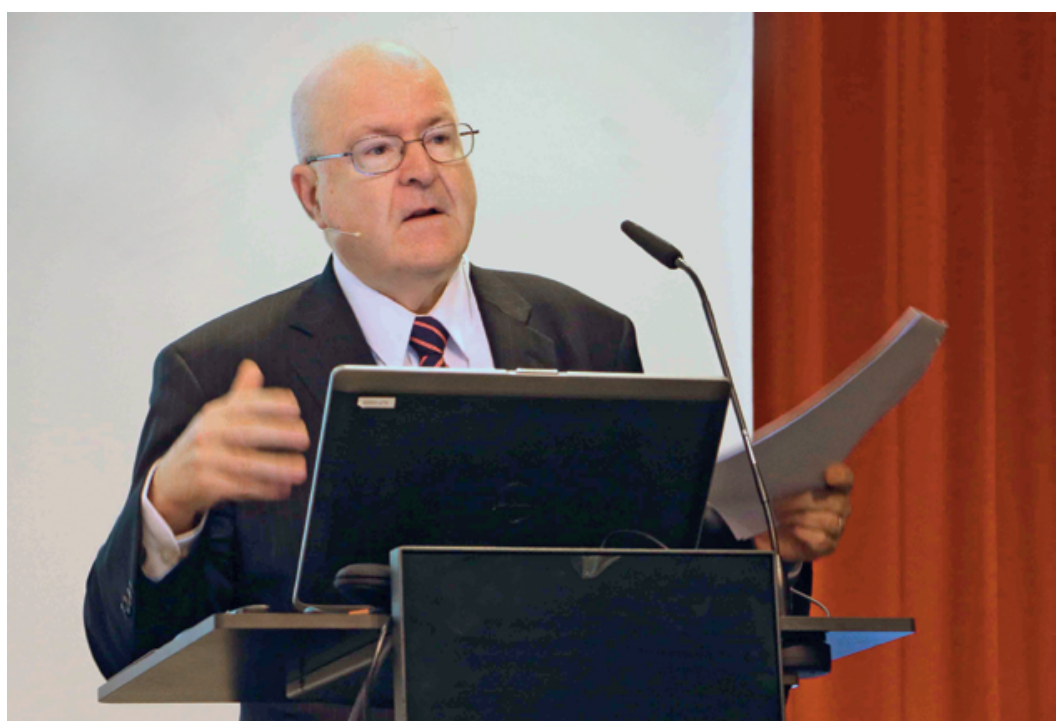

Werner Bauer, président de I'ISFM, dirige la séance plénière.

L'«accréditation 2018», qui bat actuellement son plein, constitue un véritable tour de force. Cette procédure onéreuse à plusieurs niveaux sur plusieurs années pour réévaluer tous les cursus de formation avant leur approbation par le Département fédéral de l'intérieur (DFI) exige la collaboration des sociétés de discipline, de l'ISFM, de l'Office fédéral de la santé publique (OFSP), de la Commission des professions médicales (MEBEKO), de l'Agence suisse d'accréditation et d'assurance-qualité ( $\mathrm{AAQ}$ ) et du Conseil suisse d'accréditation. Les travaux préparatoires sont désormais terminés et il s'agit maintenant de procéder aux auto-évaluations et aux expertises externes. Les premières relèvent des socié-

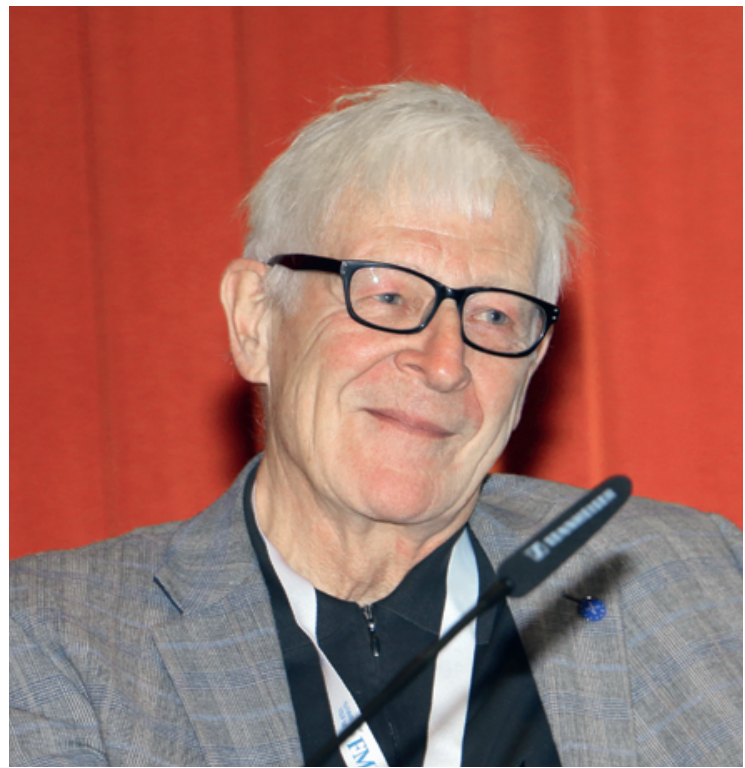

Hans Rudolf Koelz, vice-président, cesse son activité après vingt ans au service de I'ISFM. Werner Bauer lui rend hommage. tés de discipline et de l'ISFM alors que les deuxièmes, organisées par l'AAQ, se composent d'une table ronde et de rapports d'experts, sur lesquels les sociétés de discipline concernées ou l'ISFM peuvent se prononcer. Pour finir, la MEBEKO contrôle les rapports et émet pour chacun d'eux une évaluation à l'intention du DFI. Ce dernier prendra en 2018 la décision d'accorder l'accréditation.

Les activités de la plate-forme "Avenir de la formation médicale», lancée en 2010 par l'OFSP et la Conférence suisse des directrices et directeurs cantonaux de la santé (CDS) dans le but de se pencher rapidement et de manière coordonnée sur les questions que soulève la formation médicale et de proposer des propositions de solutions concrètes en concertation avec les organisations concernées, revêtent un intérêt particulier pour le corps médical. L'ISFM participe à plusieurs groupes thématiques, dont notamment un sur la "Coordination de la formation postgrade des médecins», un sujet très actuel. Ce groupe se penche sur des questions comme le nombre de spécialistes qu'il faudrait former par discipline, comment atteindre les chiffres visés et comment faire pour que les différents spécialistes formés exercent dans les régions où on a besoin d'eux. La «Focalisation de la formation médicale», un autre thème récent en cours d'évaluation, se concentre sur les possibilités de (pré-)spécialisation dans une phase précoce de la formation universitaire.

Les autres «points forts» mentionnés par Werner Bauer ont concerné le symposium MedEd, les ateliers de l'ISFM proposés avec le Royal College of Physicians, l'ISFM Award, l'encouragement de projets par l'ISFM [1] et l'Union européenne des médecins spécialistes (UEMS). Après l'immense succès rencontré par le troisième symposium MedEd, une quatrième édition sera organisée en 2017. Victimes de leur succès depuis des années, les ateliers pour médecins formateurs mis sur pied par l'ISFM en collaboration avec le très renommé Royal College of Physicians seront aussi poursuivis en 2017. Ici, a priori, pas de Brexit en vue... Seul bémol dans ce tableau réjouissant, l'encouragement de projets sera momentanément interrompu en 2017 mais devrait être repris dès que la situation financière le permettra.

\section{Le départ de Hans Rudolf Koelz}

Le point à l'ordre du jour consacré aux élections a été l'occasion de sceller la succession de Hans Rudolf Koelz, vice-président de l'ISFM, présent depuis 24 ans à toutes les séances plénières de l'ISFM. Werner Bauer a rendu un hommage poignant à ce professeur émérite en gastroentérologie et médecine interne générale en rappelant sa longévité et l'universalité de son savoir dont 


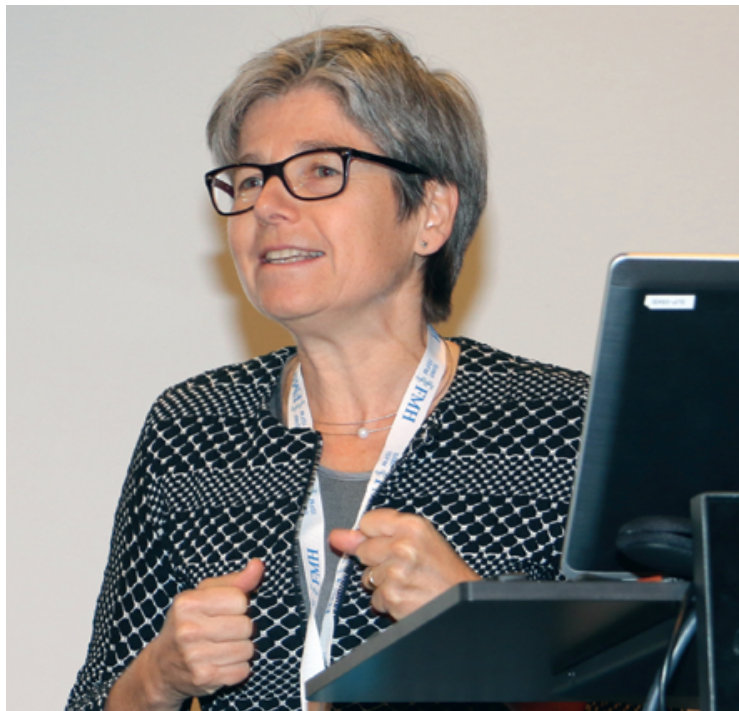

Regula Schmid a été élue vice-présidente.

l'horizon scientifique ne s'est jamais arrêté à ses spécialisations médicales. Il a accumulé des connaissances impressionnantes dans des domaines aussi divers que l'ornithologie ou l'astronomie, pour ne citer que deux exemples parmi sa vaste palette de pôles d'intérêts, et s'est aussi distingué par des prises de position «claires, voire très claires» sur les multiples et différentes questions qui ont jalonné ses mandats. Après avoir exprimé toute sa gratitude suite à cette avalanche de compliments, il a saisi l'occasion pour concéder que sa manière d'être a sans doute été assez inconfortable pour certains mais qu'elle l'a aussi été pour lui. H. R. Koelz a rappelé que le corps médical jouit de beaucoup de libertés pour concevoir les programmes de formation postgraduée. C'est très bien mais ces programmes ne sont que du papier. En définitive, c'est aux responsables de la formation de veiller à ce que les compétences médicales essentielles soient réellement vécues au quotidien. Werner Bauer a achevé son hommage par la nomination de Hans Rudolf Koelz au grade d'«Honorary Fellow of the Swiss Institute of Medical Education", une distinction non encore officielle, mais qui sait...

La succession de Hans Rudolf Koelz, qui occupait un poste à $60 \%$, sera répartie entre la Dresse Regula Schmid (20\%) et le Prof. Giatgen Spinas (40\%). Regula Schmid, spécialiste en pédiatrie et neuropédiatrie, exerce en tant que médecin adjoint à l'hôpital cantonal de Winterthur. Giatgen Spinas sera directeur de la clinique d'endocrinologie et de diabétologie, département de médecine interne, à l'hôpital universitaire de Zurich jusqu'au printemps 2017. Pendant près de vingt ans, il a œuvré dans la formation postgraduée et continue des médecins. Les deux candidats ont été élus à l'unanimité, tout comme Raphael Stolz qui s'était re-

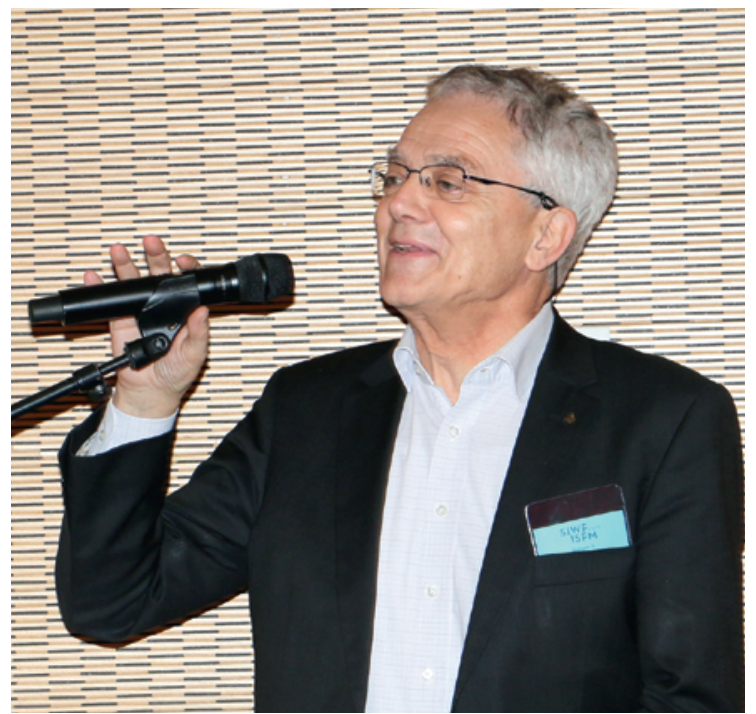

Tout comme Giatgen Spinas qui sera aussi vice-président.

présenté à la vice-présidence de l'ISFM au terme de plusieurs mandats.

\section{Logbook électronique}

Vivement débattu, le logbook électronique, une banque de données en ligne permettant aux médecins d'inscrire de manière officielle les différentes étapes de leur formation postgraduée, a suscité une riche discussion. Les informations qu'il renferme servent de base à toute demande en vue de l'octroi d'un titre de spécialiste. Dès le début, Werner Bauer précise qu'avec les connaissances d'aujourd'hui, ce projet mammouth de l'ISFM aurait été abordé différemment. Si le logbook ne peut être conçu comme un catalogue dans lequel il ne manquerait aucun des objectifs de formation à atteindre, il doit, au contraire, être convivial et simple à utiliser. Parmi les points positifs, le directeur de l'ISFM, Christoph Hänggeli, rappelle que le logbook fonctionne bien, sans support papier, et que potentiellement, il est très rapide. Dans le meilleur des cas, un dossier pourrait être traité en une journée et le titre octroyé le même jour. Aujourd'hui, tous les 83 programmes de formation ont été paramétrés et 16000 utilisateurs sont enregistrés. Jürg Jau, chef de la division ICT de la FMH, explique en détail les mesures prévues pour améliorer la plateforme. Une discussion animée s'en suit au cours de laquelle plusieurs intervenants ont exprimé leurs craintes, notamment que les simplifications prévues risquent d'entraîner la perte d'informations qualitatives importantes sur l'état de la formation. Christoph Hänggeli a pu apaiser ces craintes en signalant par exemple qu'aucune modification n'était prévue en ce qui concerne les certificats ISFM et les protocoles d'éva- 
luation. Werner Bauer a conclu en rappelant que les mesures planifiées étaient de manière générale largement acceptées, et souligné que les adaptations étaient à chaque fois mises en place en concertation avec les sociétés de discipline et que, dans la mesure du possible, leurs attentes étaient prises en compte.

\section{Budget 2017}

Après les informations relatives à la mise à jour de la plate-forme de formation continue [2], Christoph Hänggeli s'est penché sur le budget 2017 approuvé par la Chambre médicale. Celui-ci prévoit une perte de 867000 francs, à mettre principalement sur le compte des dépenses liées aux travaux nécessaires pour le logbook. Par rapport aux années précédentes, des dépenses supplémentaires importantes ont été prévues dans les domaines de l'accréditation et des coûts de personnel. Une fortune de deux à trois millions de francs est attendue au premier janvier 2018 [3].

\section{Objectifs de formation généraux et enquête de satisfaction}

Selon Werner Bauer, une tendance à davantage de poids à des "thèmes généralistes en médecine» se dessine au niveau international. Une enquête lancée en 2015 à l'initiative de l'ISFM a identifié des déficits dans les objectifs de formation généraux tels que la gestion des conflits, la communication, l'économie et le leadership. L'ISFM a alors rédigé un papier de position et il va essayer de mettre en place des offres "teach the teachers" pour les médecins cadres leur conférant un rôle clé dans l'enseignement de ces objectifs.

L'enquête de satisfaction consiste en une enquête automatisée auprès des médecins qui ont obtenu leur titre

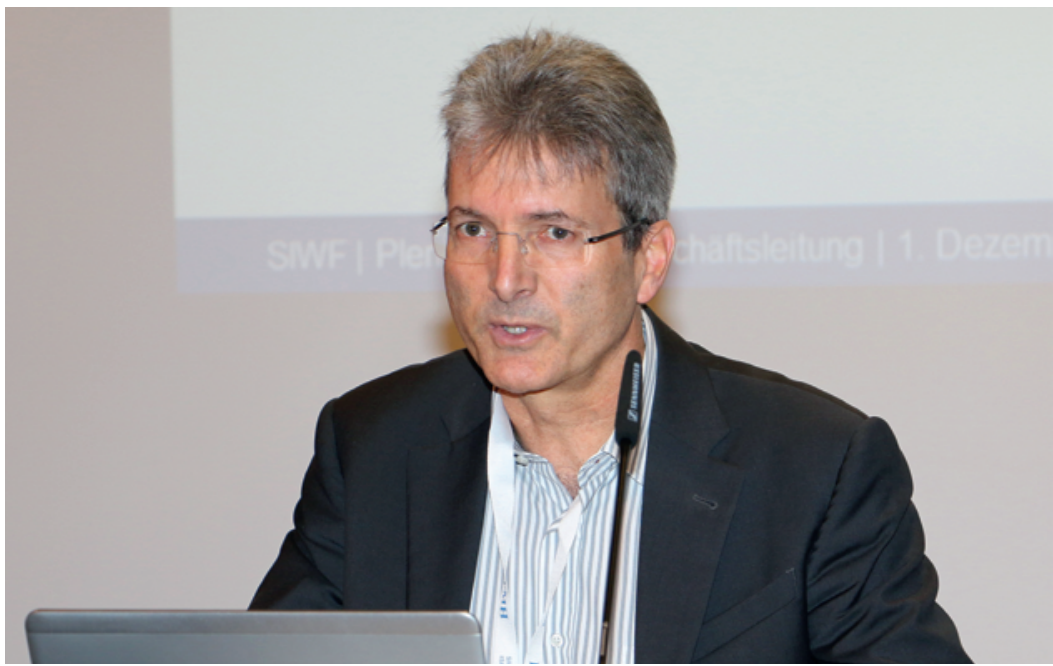

Christoph Hänggeli, directeur de I'ISFM, informe entre autres du budget 2017.

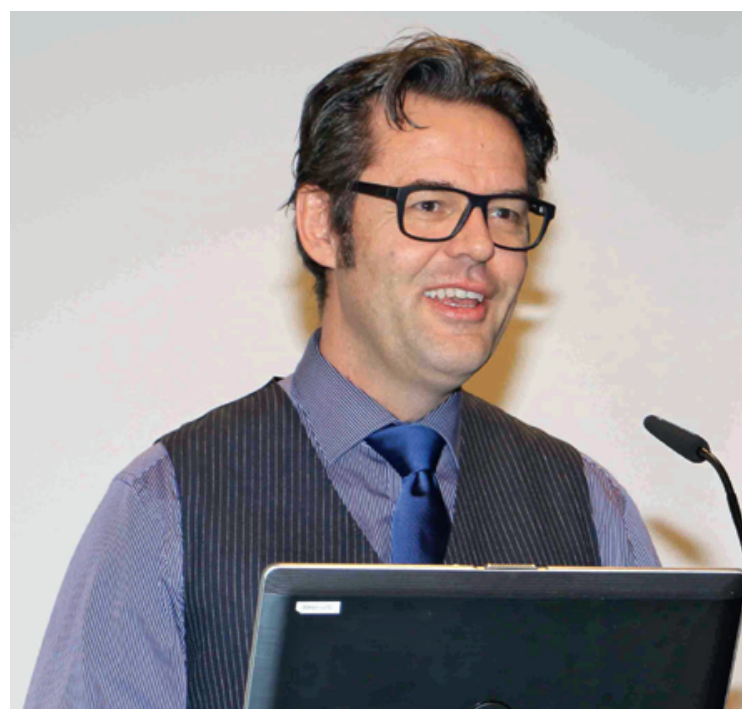

Orateur invité: Stefan Spycher de I'Office fédéral de la santé publique.

de spécialiste. Les personnes interrogées accordent une bonne note aux programmes de formation postgraduée et aux prestations du secrétariat. Elles soulignent cependant un potentiel d'amélioration en ce qui concerne le site internet de l'ISFM, ce qui sera prochainement réalisé selon Christoph Hänggeli.

\section{Planification de la main d'œuvre médicale par la formation}

Le groupe thématique "Coordination de la formation postgrade des médecins", codirigé par Werner Bauer et Stefan Spycher, se penche sur la question brûlante du pilotage de la formation médicale pré- et postgraduée. Lors d'un exposé très intéressant intitulé La planification de la main d'œuvre est-elle sensée ou insensée?, S. Spycher, vice-directeur de l'Office fédéral de la santé publique, a tout d'abord souligné la complexité de ce sujet, illustrée par des qualificatifs tels que «forte dépendance étrangère», "durée de la formation postgraduée», "offre de postes de formation", "forte densité de spécialistes vs pénurie de médecins de famille». En particulier la démographie des médecins de premier recours, dont 3,5\% part à la retraite chaque année, suscite des inquiétudes. S. Spycher rappelle que la Confédération en soi n'est pas du tout intéressée par la gestion des admissions. Cette dernière n'est devenue nécessaire que pour corriger la situation actuelle. Actuellement, on travaille sur les bases de données mais rien n'est encore décidé en ce qui concerne une «planification de la main d'œuvre». Mais si une telle planification est soigneusement mise en place avec les partenaires, elle pourrait tout à fait contribuer à la solution du problème. 


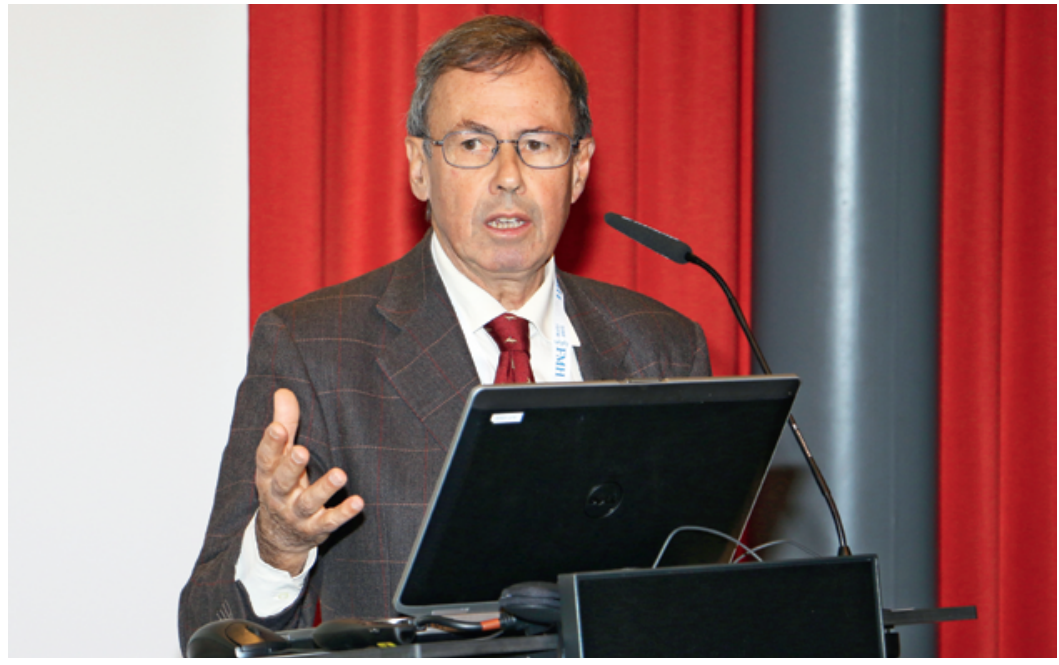

Pierre-André Michaud présente le projet «Réformer».

La présentation de Pierre-André Michaud du projet Réformer [4] a donné l'impression que la Suisse romande était plus ouverte à l'utilisation d'instruments de régulation et qu'elle avait une longueur d'avance sur la Suisse alémanique quant à la planification de la main d'œuvre. En Suisse romande, il existe déjà des réseaux qui fonctionnent bien dans plusieurs disciplines et proposent une formation structurée jusqu'à l'obtention du titre de spécialiste.

\section{Thèse versus publication}

Michael Siegrist du département Health Sciences and Technology de l'EPF Zurich a présenté les résultats 2016 de l'enquête menée chaque année auprès des médecins en formation en accordant une place particulière à la question des thèses de doctorat. Pour la majorité du corps médical, il reste important d'avoir un titre de docteur. La plupart des médecins qui n'ont pas soutenu de thèse estiment que le titre de docteur devrait être automatique une fois les études de médecine terminées alors que ceux qui ont déjà soutenu leur thèse ne sont majoritairement pas de cet avis.

En abordant la question de la «publication scientifique: condition pour un titre de spécialiste», Hans Rudolf Koelz a rappelé que le nombre de spécialistes avec un titre de docteur avait continuellement diminué ces dernières années. La réforme de Bologne y a contribué dans la mesure où elle a rehaussé les obstacles pour obtenir ce titre. De plus, depuis 2002, le titre de docteur n'est plus un préalable à l'octroi d'un titre de spécialiste, remplacé dans plusieurs disciplines par une publication scientifique. Cette tendance à la baisse du nombre de médecins ayant un titre de docteur risque médecins, estiment certains. C'est pourquoi le plénum a été mandaté avec succès pour adapter ou modifier l'article 16 de la Règlementation pour la formation postgraduée. A l'avenir, une seule publication scientifique traitant d'un thème médical ou biomédical pourra être exigée par les programmes de formation - à l'exception de cas motivés. Par ailleurs, les candidats qui ont rédigé une thèse acceptée selon les standards des universités suisses seront libérés de cette obligation.

\section{Suggestions des présidents des sociétés de discipline}

Parmi les autres points à l'ordre du jour, notons également la reconnaissance des activités de médecin militaire pour la formation postgraduée [5], les recommandations en vue du développement des examens de spécialiste, l'introduction d'examens pour les formations approfondies en psychiatrie et psychothérapie forensique pour enfants et adolescents et en psychiatrie et psychothérapie forensique pour adultes, la révision du programme de formation en radiologie et la création d'une attestation de formation complémentaire «Echographie Point of Care». Toutes ces propositions ont été clairement acceptées par les délégués.

La journée s'est terminée par l'échange désormais traditionnel avec les sociétés de discipline représentées par une Présidente (Siv Fokstuen, Société suisse de génétique médicale) et deux Présidents (Marcel Jakob, Société suisse de chirurgie, et Dominik Weishaupt, Société suisse de radiologie). Alors que la possibilité d'un dialogue critique et constructif a été largement utilisée pendant cette journée, Werner Bauer a conclu la rencontre en remerciant tous les participants pour les précieuses suggestions.

\section{Crédit photo}

Bruno Kesseli

\section{Références}

1 Le symposium MedEd 2016 a déjà fait l'objet d'un article dans le BMS présentant les lauréats de l'ISFM Award 2016 et les projets subventionnés en 2016: Kesseli B. La formation postgraduée médicale: un patient qui a de l'avenir. Bulletin des médecins suisses. 2016;97(46):1588-92. https://bullmed.ch/fr/resource/jf/journal/ file/view/article/bms.2016.05126/BMS-05126.pdf/

2 Un article à propos de la nouvelle plate-forme de formation continue est paru début 2017 dans le BMS: Hänggeli C. Avez-vous un diplôme de formation continue de l'ISFM? Bulletin des médecins suisses. 2017;98(1-2):7-11. https://bullmed.ch/fr/article/doi/ bms.2017.05280/

3 Pour de plus amples renseignements sur le budget 2017 de l'ISFM, veuillez consulter le procès-verbal de la deuxième Chambre médicale 2016: Henzen M. Procès-verbal décisionnel de la $2^{\mathrm{e}}$ Chambre médicale de 2016. Bulletin des médecins suisses. 2016;97(51-52):1758-76. https://bullmed.ch/fr/resource/jf/journal/ file/view/article/bms.2016.05227/BMS-05227.pdf/

4 Réorganisation de la formation médicale romande.

5 L’activité de médecin militaire doit pouvoir être reconnue pour autant que le programme de formation le définisse explicitement. 